mittent' behaviour, in which long periods of regular motion are interrupted by occasional chaotic 'bursts'. This is illustrated in Fig. 2, which shows the voltage in Yeh and Kao's Josephson-junction analogue as a function of time. By varying the stress parameter, in this case the amplitude of the alternating current driving the circuit, the average interval between bursts can be set to any desired value. It is difficult to conceive of a deterministic origin for intermittency, yet Manneville and Pomeau discovered ${ }^{12}$ that it is a simple property of the one-dimensional maps that govern systems such as this one. The implications for the analysis of experimental data are clear - what seems quite 'obviously' to be

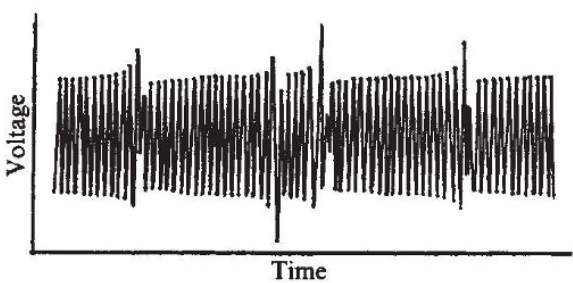

Fig. 2 Voltage as a function of time in a Josephson-junction analogue ${ }^{2}$. Nearly periodic behaviour is interrupted by occasional chaotic 'bursts'.

stochastic behaviour may arise from deterministic dynamics.

Nature contains many far-fromequilibrium systems that are liable to exhibit chaos. Some of these will be understood through models whose complexity and universal character are comparable to those of one-dimensional maps. There are also chaotic phenomena, such as fully developed hydrodynamical turbulence, that are not likely to be understood by any simple mathematical model. Yet the fact that one-dimensional maps may be adequate to describe such diverse phenomena as cardiac dysrhythmia ${ }^{13}$ and chaos in the Mixmaster Universe ${ }^{14}$ suggests that the heart of chaos is mathematically accessible. The intriguing question that remains to be answered is, how much of our chaotic world is dominated by the simple universal varieties of chaos?

Alan Wolf is in the Department of Physics, University of Texas, Austin, Texas 78712.

\footnotetext{
1. Shaw, R. The Dripping Faucet as a Model Dynamical System (Univ. California preprint, Santa Cruz, 1983)

2. Yeh, W.J.\& Kao, Y.H. Appl. Phys. Lett. 42(3), 299 (1983); Phys. Rev. Lett. 49(26), 1888 (1982).

3. Metropolis, N., Stein, M.L. \& Stein, P.R. J. combinatorial Theory A 15, 25 (1973).

4. Feigenbaum, M.J. J. Stat. Phys. 19, 25 (1978).

5. Crutchfield, J.P., Farmer, J.D. \& Huberman, B.A. Phys. Rep. 92(2), I (1982)

6. Wolf, A. \& Swift, J. Phys. Lett. 83A(5), 184 (1981)

7. Lorenz, E.N. J. atmos. Sci. 20, 130 (1963).

8. Testa, J., Perez, J. \& Jeffries, C. Phys. Rev. Lett. 48(11), 714 (1982)

9. Gibbs, H.M., Hopf, F.A., Kaplan, D.L. \& Shoemaker, R.I. Phys. Rev. Lett. 46(7), 474 (1981).

10. Maurer, \& Libchaber, A. J. Phys. (Paris) Lett. 40, L.419 (1979).

11. Simoyi, R.H., Wolf, A. \& Swinney, H.L.. Phys. Rev. Let 49(4), 245 (1982).

12. Manneville, P. \& Pomeau, Y. Physica 1D, 219 (1980).

13. Guevara, \& Glass, L. J. math. Biol. 14, I (1982).

13. Guevara, \& Glass, L. J. math. Biol. 14, I
14. Barrow, J.D. Phys. Rep. 85(1), 1 (1982).
}

\section{Nonlinear dynamics Chaos in complicated systems}

\author{
from Arun $V$. Holden
}

THE current entrancement with chaos ${ }^{1}$ began with the realization that extremely simple nonlinear systems could, with appropriate values of parameters, have irregular complicated solutions. As discussed in the previous article, solutions often change from being periodic to being chaotic in a stereotyped way ${ }^{2}$. Such powerful results, where complicated nonperiodic behaviour is produced in a simple way, lead to the hope that some of the irregularities of nature - the babbling of a brook or the mindless fluttering of a butterfly - may also be chaotic, and explicable by simple nonlinear systems with few degrees of freedom. But relating experimental observations to the chaotic limit sets of mathematical models is speculative. A tentative step is to identify and characterize irregular data as if they formed a sample solution of a deterministic system in a chaotic domain. Methods for doing this, and their applications, were considered at a recent workshop*.

An irregular experimental time series may be treated as if it were chaotic if it is possible to reconstruct an attractor of low fractal dimension from the data: this may be done graphically by plotting the delayed time series against itself ${ }^{3}$. Methods exist for estimating the fractal dimension of the reconstructed attractor $^{4}$, and for estimating the rate of divergence of neighbouring trajectories ${ }^{5}$ : it is not only possible to identify chaotic activity, but to measure how chaotic it is.

A different approach is to construct a simple nonlinear map that can be used to simulate some aspects of the experimental data: an example is to plot the peak amplitude of one oscillation against the peak amplitude of the preceding oscillation ${ }^{6}$. Another example is a Poincaire first return map, where the value a trajectory has on passing through a plane perpendicular to the trajectory is plotted against the value it has the next time it passes through that plane. These approaches are less demanding on computer time, and provide a working model, rather than quantitative measures, of chaotic activity.

Since chaotic solutions can be generated by simple nonlinear systems, it is natural to look for examples of chaotic activity in simple physical systems such as a dripping faucet. It is also possible to look for chaotic activity in complicated systems - the most complicated systems we know are living.

The heart may be considered to act as a nonlinear oscillator, so perhaps some cardiac dysfunctions correspond to chaotic behaviour ${ }^{7}$. Glass et al. ${ }^{8}$ have examined the response of endogenously active aggregates of embryonic cardiac muscle cells to periodic stimulation. An accurate

*The NATO Advanced Research Worhshop on 'Testing Nonlinear Dynamics' was held on 6-9 June 1983 description of such an aggregate is complicated, as not only are there many voltagedependent conductances in cardiac membrane, but there is also the probability of activity-dependent changes in the ionic concentrations in restricted extracellular spaces. However, the dynamic behaviour during repetitive stimulation is simple - as the frequency is increased there is a sequence of period-doubling bifurcations into a region of aperiodic chaotic activity. The experimentally determined Poincairé return maps may be iterated, to simulate the response to periodic stimulation, or since the bifurcation sequence for singlepeaked one-parameter maps of an interval into itself is largely independent of the detailed form of the map, a simplified caricature of the map can be analysed. Thus a periodically driven complicated system that exhibits chaotic activity can be represented by a simple nonlinear mapping.

Periodically excited nerve cells can also show chaotic behaviour ${ }^{9}$ - what is of more interest is whether neurones ${ }^{10}$ or neural systems can generate chaotic activity endogenously. Such chaotic activity might be pathological, or might form part of the normal irregular background activity of the nervous system. Methods now exist for investigating these possibilities.

Although no manufactured system approaches the complexity of biological systems, large-scale integration techniques have produced a generation of high-density complicated electronic control systems. Such computing devices are deterministic finite-state automata, and so it is natural to assume that their behaviour is fully predictable. Any irregularity in performance is usually ascribed to poor programming or to component failure. But complicated as well as simple nonlinear systems may exhibit chaotic solutions. Chaotic activity in a laboratory computer system may be inconvenient; in a locally autonomous complicated military system it could be unfortunate ${ }^{11}$. One can only hope that as the complexity of a system increases, access to any domains of chaotic activity becomes more and more unlikely.

Arun $V$. Holden is a lecturer at the Departmen of Physiology, University of Leeds, and an editor of Physica D - Nonlinear Phenomena.

\footnotetext{
Proc. 1982 L.us Alamos Conf. on 'Order in Chaos' Physice $7 \mathrm{D}$ (in the press)

2. Feigenbaum, M.J.J. stat. Phys. 19, 25 (1978)

3. Geisel, T. Nature 298, 322 (1982)

4. Froehling, H. et al. Physica 3D, 605 (1981).

. Bennetin, G. et al. Meccanicu 15, 9 (1980).

Loren $\angle$, E.N. J. atmos. Sci. 20, 130 (1963)

7. Keener, J.P. AMS Lect, appl. Math. 19, 299 (1981).

Glass, L. et al. Physica D (in the press).

Hayashi, H. et al. Phys. Lett. 88A, 435 (1982).

0. Holden, A.V. et al. Biol. Cybernetics 43, i 69 (1982)

11. Rapp, P. in Proc. 2nd int. Workshop on Molecular Electronic Devices (ed. Carter, F.L.) (Naval Res, L.ab.
} Washington, in the press). 